\title{
Instilação de formalina endoluminal como opção terapêutica da retite actínica hemorrágica
}

\author{
Endoluminal formalin instillation as a therapeutic option \\ of radiation-induced hemorrhagic proctitis
}

\author{
FÁBIO VIEIRA TEIXEIRA ${ }^{1}$, RAFAEL DENADAI ${ }^{2}$, RAFAEL ALICEDA FERRAZ², \\ RICARDO DE ÁLVARES GOULART ${ }^{3}$, ROGÉRIO SAAD-HOSSNE ${ }^{4}$
}

\begin{abstract}
${ }^{1}$ Professor Convidado Doutor do Departamento de Cirurgia e Ortopedia da Faculdade de Medicina da Universidade Estadual Paulista (Unesp) - Botucatu (SP); Médico da Unigastro e da Clínica Gastrosaude - Marília (SP), Brasil. ${ }^{2}$ Acadêmicos da Faculdade de Medicina da Universidade de Marília-Marília (SP), Brasil. ${ }^{3}$ Médico do Serviço de Endoscopia, Cirurgia do Aparelho Digestivo e Coloproctologia - Unigastro da Associação Beneficente Hospital Universitário (ABHU) e da Clínica Gastrosaude-Marília (SP), Brasil. ${ }^{4}$ Titular da Sociedade Brasileira de Coloproctologia; Professor-Assistente Doutor do Departamento de Cirurgia e Ortopedia da Faculdade de Medicina da Universidade Estadual Paulista (Unesp) - Botucatu (SP), Brasil.
\end{abstract}

TEIXEIRA FV, DENADAI R, FERRAZ RA, GOULART RÁ, SAAD-HOSSNE R. Instilação de formalina endoluminal como opção terapêutica da retite actínica hemorrágica. Rev bras Coloproct, 2011;31(1): 32-38.

RESUMO: A retite actínica hemorrágica é um quadro grave que pode ocorrer em qualquer paciente submetido à radioterapia pélvica, por vezes, sendo necessária terapia transfusional e internação hospitalar. A abordagem terapêutica ainda é bastante controversa. Tanto para o tratamento inicial como para casos refratários, uma das opções é a aplicação de formalina. Este método é barato, facilmente disponível, de simples execução e eficaz no controle da hemorragia. Os autores relatam dois casos de retite actínica hemorrágica de pacientes tratados com instilação de formalina endoluminal, e sua eficácia terapêutica e complicações são discutidas.

Palavras-chave: proctologia; colonoscopia; resultado de tratamento; formaldeído.

\section{INTRODUÇÃO}

A radioterapia pélvica é um método frequentemente utilizado no tratamento das neoplasias pélvicas, principalmente no câncer prostático, cervicouterino e retal, e que conta com benefícios estabelecidos ${ }^{1-5}$. No entanto, não é isenta de complicações ${ }^{3,5,6}$. O reto é o órgão mais comumente lesado após a irradiação pélvica ${ }^{3,4,7-10}$, devido à sua posição fixa e sua relação anatômica com os sítiosalvo do tratamento $0^{2-4,6,7,10-12}$.
O desenvolvimento da retite actínica é diretamente relacionado com a dose, o volume e o intervalo de radiação $0^{5,7,8}$, podendo ser classificada como aguda ou crônica ${ }^{5,7,13,14}$. A fase aguda, geralmente, é autolimitada, e os principais sintomas são diarreia, náuseas e dor abdominal, e pode permanecer até três meses ${ }^{5,10,14,15}$. Já a forma crônica, também denominada de retite actínica crônica ${ }^{14,15}$, ocorre em até $20 \%$ dos pacientes ${ }^{5,7,14,16}$, normalmente após meses ou anos à irradiação $0^{5,13-15}$. Este quadro de retite actínica crônica é resultante da endarterite obliterante, fibrose da sub-

Trabalho realizado no Serviço de Endoscopia, Cirurgia do Aparelho Digestivo e Coloproctologia - UNIGASTRO, Associação Beneficente Hospital Universitário (ABHU), Marília (SP), Brasil.

Conflito de interesse: nada a declarar.

Recebido em: 25/06/2010

Aprovado em: 14/04/2011 
mucosa e formação de novos vasos ${ }^{8,10,13,14,16}$, com consequente isquemia do tecido retal ${ }^{10,13}$, o que determina friabilidade da mucosa, sangramento, úlceras, estenoses e fístulas ${ }^{13}$. Clinicamente, apresenta-se com uma variedade de sintomas, incluindo diarreia mucoide, tenesmo, dor e sangramento ${ }^{5,7,13,14,16}$.

O sangramento pode ser mínimo ou, em alguns casos, maciço, determinando a retite actínica hemorrágica $(\mathrm{RAH})$, que é geralmente grave, implicando em repetidas transfusões e internação hospitalar2-5,11,13,15-18, constituindo um grande desafio terapêutico ${ }^{3,4,9,11,15}$.

O tratamento da RAH ainda é controverso, com diferentes ${ }^{1,6,7,11}$ modalidades disponíveis, sendo $5,7,8,13,14,19$ difícil indicar ou escolher qualquer terapia baseada em evidências ${ }^{5,7,8,14,19}$, devido à ausência de ensaios clínicos randomizados e controlados ${ }^{7,8,19}$.

Neste contexto, uma boa opção terapêutica é a aplicação de formalina, método simples, barato, disponível em todos os hospitais ${ }^{1-4,6,7,9,10,13,15,17}$ e eficaz tanto como primeira opção terapêutica ${ }^{6,9,10}$, quanto nos casos refratários de $\mathrm{RAH}^{1-4,11-13,15,16,20,21}$.

A irrigação do reto com formalina a $4 \%$ para o tratamento da RAH vem sendo realizada com sucesso no Serviço de Endoscopia, Cirurgia do Aparelho Digestivo e Coloproctologia - UNIGASTRO, desde $2002^{22}$. Em virtude do exposto, é oportuno relatar dois casos de RAH, de difícil controle, tratados com a instilação endoluminal de formalina a 4 e $2 \%$, para reforçar a experiência dos autores deste trabalho. Também foram discutidas sua eficácia terapêutica e complicações.

\section{RELATO DO CASO}

\section{Relato 1}

Paciente do sexo masculino, 67 anos, procurou o serviço de coloproctologia com hemorragia digestiva baixa há quatro meses. Referiu que o sangramento inicialmente era intermitente, porém, intensificou-se nas últimas semanas. Associado ao quadro, ele relatou astenia, tonturas, dores e câimbras nas pernas. Ao exame físico, apresentava-se descorado $(+++/+4)$, com as extremidades frias e taquicárdico.

Referia tratamento prévio em outro serviço, sem melhora do quadro hemorrágico, com necessidade de transfusão sanguínea, não sabendo quantificar o nú- mero de bolsas. Não foi possível a obtenção do relatório médico sobre o procedimento realizado na tentativa de cessar o sangramento.

Como antecedentes pessoais, o paciente relatou ter sido submetido à radioterapia pélvica há 12 meses como terapia coadjuvante, no tratamento do câncer de próstata.

O paciente foi internado para estabilização hemodinâmica e o resultado do hemograma de entrada foi: $\mathrm{Hb}=7,6 \mathrm{~g} / \mathrm{dL} ; \mathrm{HT}=29,1 \%$; $\mathrm{VCM}=74,915 \mathrm{fle}$ $\mathrm{HCM}=23,982 \mathrm{pg}$.

Foi indicada a retossigmoidoscopia, que evidenciou, a partir de $2 \mathrm{~cm}$ acima da linha pectínea, a presença de mucosa friável ao toque do aparelho, com sangramento recente e presença de coágulos (Figura $1 \mathrm{~A}$ e B), portanto, concluiu-se tratar de RAH com sangramento ativo.

Em virtude da gravidade visualizada pela retossigmoidoscopia e a necessidade de múltiplas transfusões (seis concentrados de hemácias), no quarto dia de internação hospitalar, foi proposto o tratamento com formalina a $4 \%$.

Após a concordância do paciente sobre o procedimento, e com ele em posição lateral de Sims, sob sedação com propofol, assistido por um anestesiologista, realizou-se a colonoscopia com a aplicação via retal de $500 \mathrm{~mL}$ de solução de formalina a 4\%. Foram instiladas, via sonda retal posicionada junto às lesões, alíquotas de $50 \mathrm{~mL}$ da solução de formalina. Após um minuto, aplicou-se solução fisiológica a $0,9 \%$, com o objetivo de lavar toda mucosa retal. Foram realizadas dez instilações de formalina até completar $500 \mathrm{~mL}$. $\mathrm{O}$ reto e o canal anal foram lavados abundantemente com solução salina, após o procedimento. Depois da instilação da solução, observou-se a parada completa do sangramento.

O paciente evoluiu sem nenhuma complicação e saiu de alta 24 horas pós-procedimento em uso de sulfato ferroso. No seguimento clínico manteve-se assintomático com hábito intestinal regular e fezes sem sinais de sangramento. As retossigmoidoscopias seriadas demonstraram a eficácia terapêutica e após 8 meses da aplicação, um novo exame demonstrou retite actínia em resolução, sem sinais de sangramento (Figura $1 \mathrm{C}$ e D), sendo que durante este período não foi necessário mais nenhuma transfusão sanguínea. 


\section{Relato 2}

Paciente do sexo masculino, 75 anos, encaminhado ao serviço de coloproctologia com sangramento gastrintestinal baixo há três meses. Relatou que o sangramento, inicialmente, aparecia nas evacuações, porém, nas últimas semanas, passou a sangrar ativamente pelo ânus. Concomitante ao quadro, ele relatou cefaleia e fraqueza muscular. $\mathrm{O}$ exame físico revelou palidez cutâneo-mucosa $(++/+4)$ e taquicardia. $\mathrm{O}$ paciente não recebeu tratamento anterior. Como antecedente, referiu ter sido submetido à radioterapia coadjuvante pélvica há nove meses, no tratamento do câncer de próstata. Foi internado para estabilização do estado hemodinâmico e melhor elucidação do quadro.

$\mathrm{O}$ hemograma diagnosticou anemia ferropriva $(\mathrm{Hb}=8,9 \mathrm{~g} / \mathrm{dL} ; \mathrm{HT}=32,3 \% ; \quad \mathrm{VCM}=76,915 \mathrm{fl}$ e $\mathrm{HCM}=25,744 \mathrm{pg}$ ) secundária à história de sangramento. A retossigmoidoscopia mostrou mucosa do reto até 10 $\mathrm{cm}$, com intenso processo inflamatório, hipervascularização intensa, sangramento do tipo "babação" e friabilidade ao toque do aparelho (Figura 2A e 2B). Diagnosticando-se, portanto, RAH com sangramento ativo.

Em virtude do quadro clínico e aspecto visibilizado à retossigmoidoscopia, optou-se pelo tratamento com formalina a $2 \%$. Após a concordância do paciente com o procedimento, a colonoscopia foi realizada.

Foi utilizada a mesma técnica descrita no primeiro caso, com a diferença da concentração da solução de formalina, a qual, para este procedimento, foi de $2 \%$. Assim, como no caso anterior, após a instilação da solução, observou-se a parada completa do sangramento (Figura 2C).

O segundo paciente também evoluiu sem intercorrência e recebeu alta 24 horas pós-procedimento, em uso de sulfato ferroso.

No seguimento clínico, manteve-se assintomático com hábito intestinal regular e fezes sem sinais de sangramento. A retossigmoidoscopia, após dois meses da aplicação, mostrou retite actínia em resolução, sem sinais de sangramento (Figura 2D). O paciente se encontra em seguimento com retossigmoidoscopias seriadas e, até o momento, não foi necessária transfusão sanguínea.

\section{DISCUSSÃO}

Apesar da enorme quantidade de métodos terapêuticos disponíveis, ainda não há consenso sobre qual deles é o ideal para o tratamento da $\mathrm{RAH}^{7,10}$. Diversas medidas têm sido utilizadas, incluindo a terapia oral (derivados do ácido salicílico - 5-ASA), terapias de instilação retal (corticosteroides, sucralfato ou 5-ASA), tratamento térmico (eletrocoagulação bipolar ou laser) e oxigenoterapia hiperbárica ${ }^{5,7,8-11,13-15,19}$. Porém, a maioria das opções permite somente o controle das formas leves da doença (grau I e II) ${ }^{3,12,15,16,23}$, não se mostrando eficazes em casos mais graves (grau III) e refratários $3,4,6,7,11,15,23$.

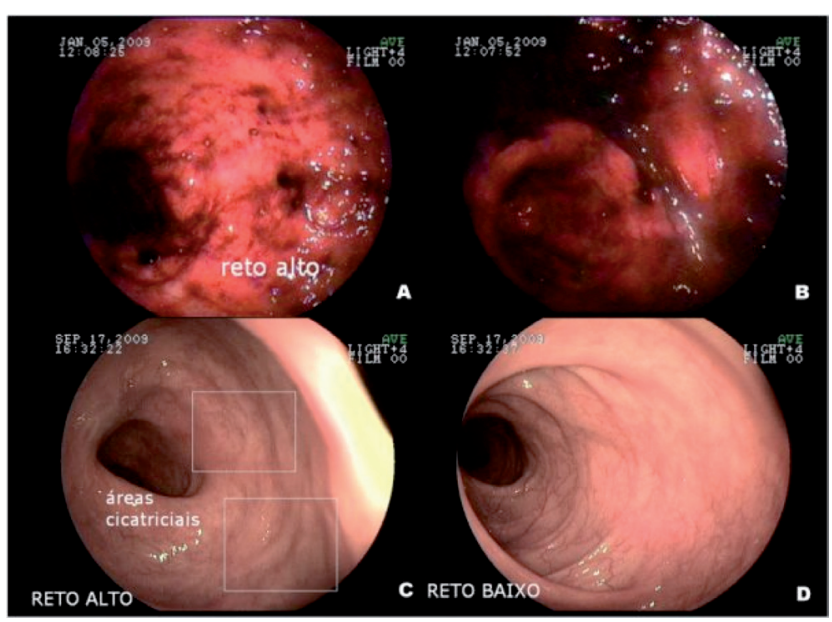

Figura 1. Retossigmoidoscopia mostrando (A e B) mucosa friável ao toque do aparelho com sinais de sangramento recente $e$ presença de coágulos e (B e C) mucosa cicatrizada sem evidências de sangramento, oito meses após aplicação de formalina a 4\%.

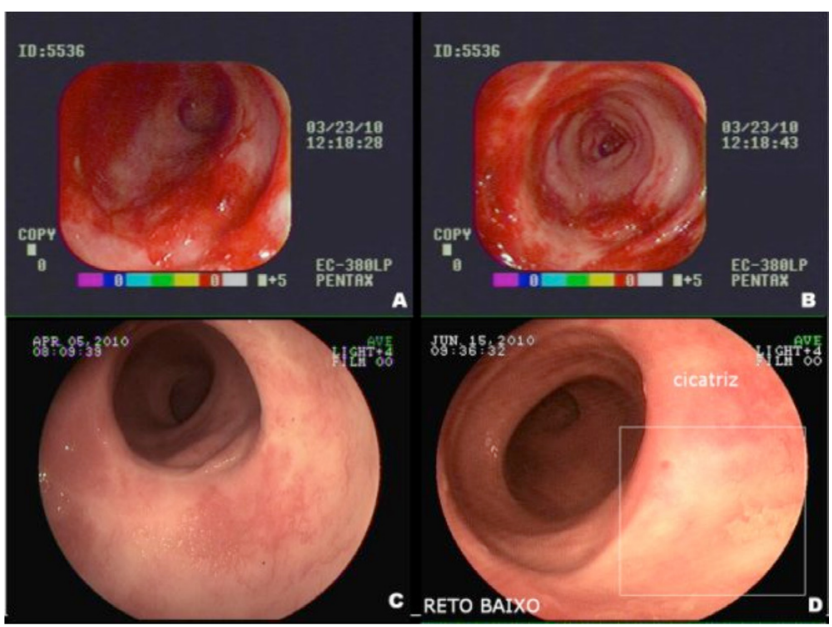

Figura 2. Retossigmoidoscopia revelando (A e B) mucosa do reto com intenso processo inflamatório, hipervascularização intensa e sangramento. Retossigmoidoscopia demonstrando (C) parada completa do sangramento após instilação da formalina a $2 \%$ aplicação e (D) retite actínica hemorrágica em resolução, dois meses após aplicação da solução. 
A ressecção do reto também pode ser considerada, mas esta é tecnicamente difícil devido à fibrose e relativa isquemia dos tecidos irradiados, podendo levar a complicações graves com alta morbidade $3,4,9,15,16$. Deve-se, portanto, considerar este procedimento somente na falha dos outros tratamentos ${ }^{3,15}$.

Assim, surgem como alternativas a aplicação de formalina ${ }^{1-4,6,7,9,10-13,15-17,20-22}$ e o tratamento térmico por coagulação com plasma de argônio $7,8,13,14,19,23,24$. Este, entretanto, é caro, não está disponível em todos os hospitais, exige treinamento específico e múltiplas sessões $^{3,4,10,12,15,23}$. Além disso, pode levar a perfurações retais em alguns casos ${ }^{2,3,15,17}$ e obter eficácia diminuída, nos casos de hemorragia intensa ${ }^{5}$.

Um estudo retrospectivo ${ }^{24}$, que comparou a eficácia da aplicação de formalina com a coagulação com plasma de argônio, mostrou que este é mais eficaz no controle da hemorragia. Contudo, os autores concluem que a gravidade da retite não foi avaliada, fato que poderia modificar a resposta em cada subgrupo.

Desta forma, a formalina é eficaz no tratamento da $\mathrm{RAH}^{1-4,6,9-13,15,16,20,21}$, especialmente nos casos graves e refratários em que outras modalidades terapêuticas não foram capazes de eliminar o sangramento $^{1-4,11-13,15,16,20,21}$. Além disso, apresenta vantagens como baixo custo, fácil disponibilidade e manipulação $0^{1-4,6,7,9,10,13,15,17}$.

A formalina tem sido eficaz em controlar a cistite hemorrágica induzida por radiação desde $1968^{4,10,14,15}$ e, a partir de 1986, passou a ser utilizada como opção no tratamento da RAH, após a publicação de Rubinstein et al. ${ }^{25}$, os quais instilaram $2 \mathrm{~L}$ de formalina $(3,6 \%)$, por 15 minutos, em um paciente com RAH irradiado para o câncer de bexiga, obtendo resolução completa do quadro. Quando em contato com a mucosa, essa substância produz cauterização química local, determinando esclerose dos vasos com consequente melhora do sangramento $6,8,11,14$.

Após esse trabalho, surgiram várias publicações ${ }^{1-}$ 4,6,9,10-12,15-18,20-24,26-32, em que os autores vêm tentando determinar a melhor técnica de aplicação, a concentração mais eficaz e identificar as complicações relacionadas com a sua aplicação. Na Tabela 1, foram agrupadas, de forma comparativa, as diferentes séries de casos.

Em relação à técnica original descrita por $\mathrm{Ru}-$ binstein et al. ${ }^{25}$, apareceram algumas variações no de- correr destes mais de 20 anos da publicação. A solução de formalina pode ser aplicada no reto basicamente de duas maneiras: por irrigação, quer de solução de formalina a $3,6^{25}, 4^{1,3,12,16,21,26}$ ou $5 \%{ }^{17}$; e uma outra técnica, proposta por Seow-Choen et al. ${ }^{27}$, é a aplicação direta com gaze embebida em formol, seja com solução a $4^{1,4,6,9-12,15,16,18,20,26-32}$ ou $10 \%{ }^{2}$. No presente trabalho, o controle da hemorragia foi atingido com a instilação endoluminal de formalina a 4 e $2 \%$.

Em ambos os casos, o procedimento foi realizado com cautela para evitar lesões cutâneas secundárias ao contato da solução de formalina com a região anal e perianal. Independente do método utilizado, a maioria dos autores recomenda a lavagem do reto com solução salina após a aplicação da formalina ${ }^{5,11,23}$. Para a proteção do períneo, podem ser aplicadas várias camadas de pomada protetoras antes do procedimento ${ }^{11,12,15,16,27}$, ou ainda um cateter de Foley pode ser introduzido e insuflado no reto para evitar qualquer fuga de formalina à mucosa anal e ao períneo ${ }^{3,5,23}$. Além disto, uma gaze pode ser colocada acima da margem de sangramento a fim de proteger a mucosa saudável ${ }^{3}$.

Mesmo com altas taxas de sucesso (acima de $90 \%)^{1-3,9,12,15,16,18,26,32}$ e havendo estudos sem nenhuma complicação ${ }^{6,9,10,15,17,20}$, os efeitos colaterais da aplicação de formalina não devem ser negligenciados ${ }^{5,11,12,14,16,17}$. Entre as principais morbidades estão: ulceração anal, estenose retal e anal, diarreia grave, febre, colite induzida por formalina, incontinência fecal e dor anal. É evidente que algumas destas complicações podem ocorrer devido à radiação inicial ${ }^{11,14}$.

Um grupo ${ }^{21}$ sugeriu que a estenose ou a incontinência anal podem estar relacionadas ao uso do afastador anal e não ao uso de formol, pois o uso da técnica com um sigmoidoscópio rígido parece não estar associado com tais alterações. Além disso, outros autores ${ }^{18}$ descreveram dois cânceres anorretais (adenocarcinoma e carcinoma espinocelular) após tratamento com formalina; os dois pacientes haviam recebido radioterapia como forma de tratamento de um adenocarcinoma prostático. Porém, os autores concluíram que é difícil determinar o papel da aplicação da formalina nestes casos e que qualquer ulceração ou lesão suspeita deve ser tratada com seriedade e propedêutica adequada, e não simplesmente atribuí-las a mudanças crônicas secundárias à radiação. 


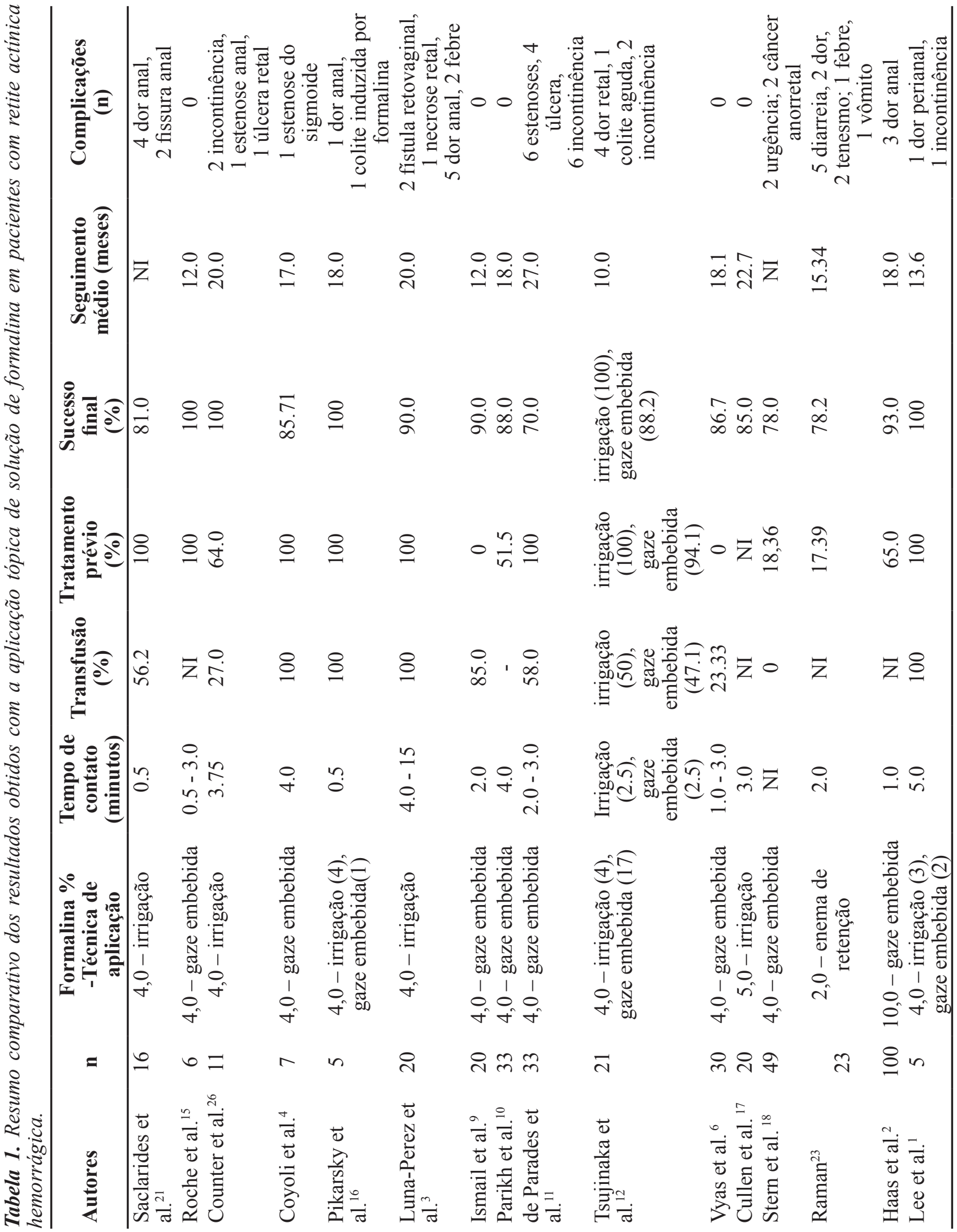




\section{CONCLUSÃO}

Em suma, mesmo havendo várias modalidades para o tratamento da RAH, não existe ainda uma abordagem ideal. Sendo assim, a aplicação endoluminal de formali- na, por ser de baixo custo, fácil acesso e manuseio, deve ser considerada na terapêutica inicial ou em casos refratários de RAH. Não deve-se, contudo, esquecer de suas prováveis morbidades, sendo necessários mais estudos para melhorar sua eficácia e reduzir tais complicações.

\begin{abstract}
The radiation-induced hemorrhagic proctitis is a serious condition that can occur in any patient undergoing pelvic radiotherapy, sometimes being required hospitalization and transfusion therapy. The therapeutic approach is still very controversial. Both for the initial treatment and for refractory cases, one option is formalin application. This method is cheap, easily available, simple to perform, and effective in controlling bleeding. The authors report two cases of radiation-induced hemorrhagic proctitis treated with endoluminal instillation of formalin, and they discuss its therapeutic efficacy and complications.
\end{abstract}

Keywords: proctology; colonoscopy; treatment outcome; formaldehyde.

\section{REFERÊNCIAS}

1. Lee SI, Park YA, Sohn SK. Formalin application for the treatment of radiation-induced hemorrhagic proctitis. Yonsei Med J 2007;48(1):97-100.

2. Haas EM, Bailey HR, Farragher I. Application of 10 percent formalin for the treatment of radiation-induced hemorrhagic proctitis. Dis Colon Rectum 2007;50(2):213-7.

3. Luna-Pérez P, Rodríguez-Ramírez SE. Formalin instillation for refractory radiation-induced hemorrhagic proctitis. J Surg Oncol 2002;80(1):41-4.

4. Coyoli-García O, Alvarado-Cerna R, Corona Bautista A, Pacheco Pérez M. The treatment of rectorrhagia secondary to postradiation proctitis with $4 \%$ formalin. Ginecol Obstet Mex 1999;67:341-5.

5. de Parades V, Bauer P, Marteau P, Chauveinc L, Bouillet T, Atienza P. Non surgical treatment of chronic hemorrhagic radiation rectitis. Gastroenterol Clin Biol 2007;31(11):91928.

6. Vyas FL, Mathai V, Selvamani B, John S, Banerjee Jesudason SR. Endoluminal formalin application for haemorrhagic radiation proctitis. Colorectal Dis 2006;8(4):342-6.

7. Cotti G, Seid V, Araujo S, Souza AH Jr, Kiss DR, Habr-Gama A. Conservative therapies for hemorrhagic radiation proctitis: a review. Rev Hosp Clin Fac Med Sao Paulo 2003;58(5):28492.

8. Denton AS, Andreyev HJ, Forbes A, Maher EJ. Systematic review for non-surgical interventions for the management of late radiation proctitis. Br J Cancer 2002;87(2):134-43.

9. Ismail MA, Qureshi MA. Formalin dab for haemorrhagic radiation proctitis. Ann R Coll Surg Engl 2002;84(4):263-4.

10. Parikh S, Hughes C, Salvati EP, Eisenstat T, Oliver G, Chinn $\mathrm{B}$, et al. Treatment of hemorrhagic radiation proctitis with 4 percent formalin. Dis Colon Rectum 2003;46(5):596-600.

11. de Parades V, Etienney I, Bauer P, Bourguignon J, Meary N, Mory B, et al. Formalin application in the treatment of chronic radiation-induced hemorrhagic proctitis - an effective but not risk-free procedure: a prospective study of 33 patients. Dis Colon Rectum 2005;48(8):1535-41.

12. Tsujinaka S, Baig MK, Gornev R, de la Garza C, Hwang JK, Sands D, et al. Formalin instillation for hemorrhagic radiation proctitis. Surg Innov 2005;12(2):123-8.

13. Sharma B, Pandey D, Chauhan V, Gupta D, Mokta J, Thakur SS. Radiation Proctitis. JIACM 2005;6(2):146-51.

14. Leiper K, Morris AI. Treatment of radiation proctitis. Clin Oncol (R Coll Radiol) 2007;19(9):724-9.

15. Roche B, Chautems R, Marti MC. Application of formaldehyde for treatment of hemorrhagic radiation-induced proctitis. World J Surg 1996;20(8):1092-4.

16. Pikarsky AJ, Belin B, Efron J, Weiss EG, Nogueras JJ, Wexner SD. Complications following formalin installation in the treatment of radiation induced proctitis. Int J Colorectal Dis 2000;15(2):96-9.

17. Cullen SN, Frenz M, Mee A. Treatment of haemorrhagic radiation-induced proctopathy using small volume topical formalin instillation. Aliment Pharmacol Ther 2006;23(11):1575-9.

18. Stern DR, Steinhagen RM. Anorectal cancer following topical formalin application for haemorrhagic radiation proctitis. Colorectal Dis 2007;9(3):275-8.

19. Denton A, Forbes A, Andreyev J, Maher EJ. Non surgical interventions for late radiation proctitis in patients who have received radical radiotherapy to the pelvis. Cochrane Database Syst Rev 2002;(1):CD003455.

20. Biswal BM, Lal P, Rath GK, Shukla NK, Mohanti BK, Deo S. Intrarectal formalin application, an effective treatment for grade III haemorrhagic radiation proctitis. Radiother Oncol 1995;35(3):212-5.

21. Saclarides TJ, King DG, Franklin JL, Doolas A. Formalin instillation for refractory radiation-induced hemorrhagic proctitis. Report of 16 patients. Dis Colon Rectum 1996;39(2):196-9. 
22. Teixeira FV, Pilon B, Marchioni R. Tratamento da retite actínica hemorrágica com o uso de solução de formalina intraretal: Relato de caso. Rev Bras Coloproct 2002;22(3):184-9.

23. Raman RR. Two percent formalin retention enemas for hemorrhagic radiation proctitis: a preliminary report. Dis Colon Rectum 2007;50(7):1032-9.

24. Alfadhli AA, Alazmi WM, Ponich T, Howard JM, Prokopiw I, Alaqeel A, et al. Efficacy of argon plasma coagulation compared to topical formalin application for chronic radiation proctopathy. Can J Gastroenterol 2008;22(2):129-32.

25. Rubinstein E, Ibsen T, Rasmussen RB, Reimer E, Sørensen BL. Formalin treatment of radiation-induced hemorrhagic proctitis. Am J Gastroenterol 1986;81(1):44-5.

26. Counter SF, Froese DP, Hart MJ. Prospective evaluation of formalin therapy for radiation proctitis. Am J Surg 1999;177(5):396-8.

27. Seow-Choen F, Goh HS, Eu KW, Ho YH, Tay SK. A simple and effective treatment for hemorrhagic radiation proctitis using formalin. Dis Colon Rectum 1993;36(2):135-8.

28. Isenberg GA, Goldstein SD, Resnik AM. Formalin therapy for radiation proctitis. JAMA 1994;272(23):1822.
29. Mathai V, Seow-Choen F. Endoluminal formalin therapy for haemorrhagic radiation proctitis. Br J Surg 1995;82(2):190.

30. Chapuis P, Dent O, Bokey E, Galt E, Zelas P, Nicholls M, et al. The development of a treatment protocol for patients with chronic radiation-induced rectal bleeding. Aust N Z J Surg 1996;66(10):680-5.

31. Goldinger M. Radiation-induced hemorrhagic proctitis treated with formalin. A simple and efficient help against adverse effects of radiation. Lakartidningen 1998;95(11):1142-3.

32. Yegappan M, Ho YH, Nyam D, Leong A, Eu KW, Seow C. The surgical management of colorectal complications from irradiation for carcinoma of the cervix. Ann Acad Med Singapore 1998;27(5):627-30.

\section{Endereço para correspondência:}

\section{FÁBIO VIEIRA TEIXEIRA}

Serviço de Endoscopia, Cirurgia do Aparelho Digestivo e Coloproctologia - UNIGASTRO

Rua Doutor Próspero Cecílio Coimbra, 80 - Cidade Universitária CEP: 17525-160 - Marília (SP), Brasil

E-mail fabioteixeira@unimedmarilia.com.br 